\title{
Importância da perimetria manual cinética na avaliação de pacientes com glaucoma avançado
}

\author{
Manual kinetic perimetry value to monitor \\ advanced glaucomatous patients
}

Nadia Ajub Moysés ${ }^{1}$ Elaine Caetano de Souza² ${ }^{2}$ Carlos Akira Omi³

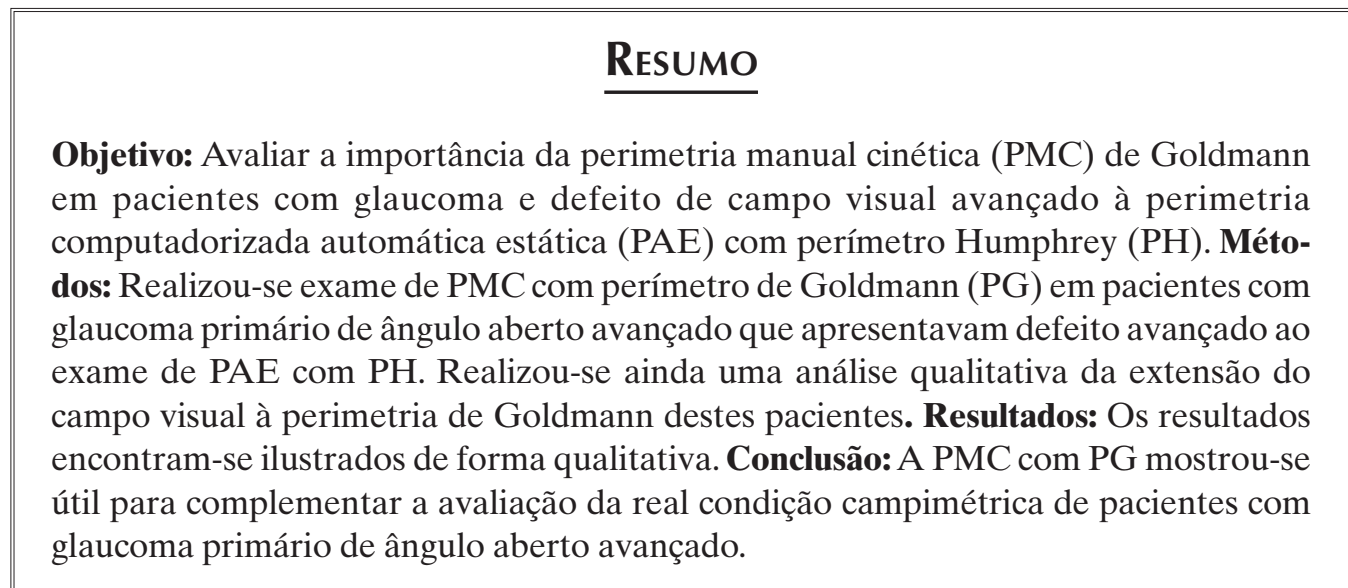

Descritores: Perimetria/métodos; Cinética; Glaucoma de ângulo aberto; Campos visuais

\footnotetext{
${ }^{1}$ Médica assistente do Setor de Glaucoma do Hospital de Transplantes do Estado de São Paulo Dr. Euryclides de Jesus Zerbini - São Paulo - (SP), Brasil; Médica do Setor de Glaucoma do Instituto Paulista de Oftalmologia Especializada - São Paulo - (SP), Brasil; ${ }^{2}$ Ortoptista e Pós-Graduação em Ciências Visuais pela Universidade Federal de São Paulo - UNIFESP - São Paulo (SP), Brasil; ${ }^{3}$ Doutor, Membro do Setor de Glaucoma da Universidade Federal de São Paulo - UNIFESP - São Paulo - (SP), Brasil; Responsável pelo Setor de Glaucoma do Instituto Paulista de Oftalmologia Especializada - São Paulo - (SP), Brasil.

Trabalho desenvolvido no Instituto Paulista de Oftalmologia Especializada - São Paulo (SP) - Brasil.
} 


\section{INTRODUÇÃO}

A pesar do amplo uso da perimetria computadorizada automática estática (PAE) no diagnóstico e acompanhamento do glaucoma, a perimetria manual cinética ainda possui papel importante em alguns pacientes ${ }^{(1)}$.

A perimetria cinética é considerada método importante nos casos em que há a necessidade de se definir os padrões e limites de escotomas avançados ${ }^{(2)}$.O chamado fenômeno de dissociação estático-cinético ou fenômeno de Riddoch considera que há uma diferença entre a percepção de um estímulo móvel e de um estático $^{(3)}$. A sensibilidade retiniana para um estímulo móvel é maior que a para um estático ${ }^{(4)}$. Um estímulo móvel consegue detectar os limites de um escotoma de forma mais precisa que um estímulo estático. Além disso, um teste com estímulos em movimento assemelha-se mais com a vida real do que um com estímulos estáticos ${ }^{(5)}$.

A perimetria manual permite um maior controle de perdas de fixação, devido à maior interação entre 0 examinador e o paciente durante o exame ${ }^{(6)}$. Documentou-se uma preferência significativa pelo paciente com perda de campo visual avançada pela PMC em comparação com a automática estática ${ }^{(7,8)}$. Pacientes com uma importante redução da visão central, desordens cognitivas ou dificuldade de cooperação na PAE, podem realizar um exame mais confiável através da PMC de Goldmann ${ }^{(9)}$.

O objetivo deste trabalho é avaliar a importância da perimetria manual cinética (PMC) de Goldmann em pacientes com glaucoma e defeito de campo visual avançado à da perimetria computadorizada automática estática (PAE) com perímetro de Humphrey.

\section{MéTOdos}

Este estudo transversal foi realizado no Instituto Paulista de Oftalmologia Especializada, São Paulo Brasil, no período de dezembro de 2008 a abril de 2009. Selecionaram-se pacientes com diagnóstico de glaucoma de ângulo aberto e em fase avançada. Os critérios de inclusão neste estudo foram escavação do nervo óptico igual ou maior que 0,9 , avaliada pelo mesmo oftalmologista especialista em glaucoma; e presença de dano campimétrico avançado e reprodutível, característico desta fase da doença ao exame de perimetria estática automática com perímetro de Humphrey (modelo 750, Humphrey Intruments Carl Zeiss), programa 24-2 com estratégia Full Threshold SITA.
O exame de campimetria manual cinética foi realizado no perímetro de Goldmann. $\mathrm{O}$ aparelho foi calibrado antes da realização de cada exame. Os estímulos escolhidos foram V4e, III4e, I4e, I3e e I2e e apresentados de forma cinética com intervalos de 15 graus, até o menor estímulo percebido pelo paciente. Além disso, pesquisouse a presença de escotomas de forma estática até 15 graus centrais do campo visual, sempre que possível.

Todos os pacientes usavam a refração adequada e atualizada durante a realização dos exames de campo visual e esses foram realizados por um mesmo examinador. Na campimetria cinética de Goldmann a refração foi utilizada na avaliação dos 30 graus centrais.

Os pacientes assinaram o Termo de Consentimento livre e esclarecido e o trabalho foi aprovado pelo Comitê de Ética em Pesquisa Médica do Hospital Santa Cruz.

Realizou-se uma análise qualitativa da extensão do campo visual à perimetria de Goldmann destes pacientes.

\section{Resultados}

Incluiu-se no estudo 7 pacientes ( 6 homens e 1 mulher), em um total de 7 olhos, com idade entre 61 e 85 . A acuidade visual dos pacientes variou de 20/25 a 20/200.

Analisou-se a extensão do campo visual realizado com o perímetro de Goldmann (PG) dos sete olhos estudados. Os resultados obtidos à $\mathrm{PG}$ assim como o respectivo exame realizado no perímetro de Humprey encontram-se ilustrados abaixo.

\section{Dıscussão}

Os parâmetros mais utilizados no acompanhamento da evolução do glaucoma primário de ângulo aberto são pressão intraocular, escavação do disco óptico e avaliação do campo visual. Nos casos de dano glaucomatoso avançado, as alterações morfológicas no nervo óptico são difíceis de avaliar pois estão no limite para medida e observação de sua progressão. Nestes casos, a perimetria assume um papel importante considerando-se que uma discreta alteração morfológica resulta em uma visível alteração campimétrica ${ }^{(8)}$.

A PAE parece ser mais sensível que a PMC na deteç̧ão da perda glaucomatosa de campo visual ${ }^{(10-12)}$. No entanto, estes estudos enfatizaram casos iniciais de perda glaucomatosa do campo visual. Em casos de perda avançada de campo visual o uso da perimetria limiar estática padrão, que testa a sensibilidade bem próxima ao limiar, pode ser ineficiente, demorada e cansativa ao paciente ${ }^{(8)}$. 

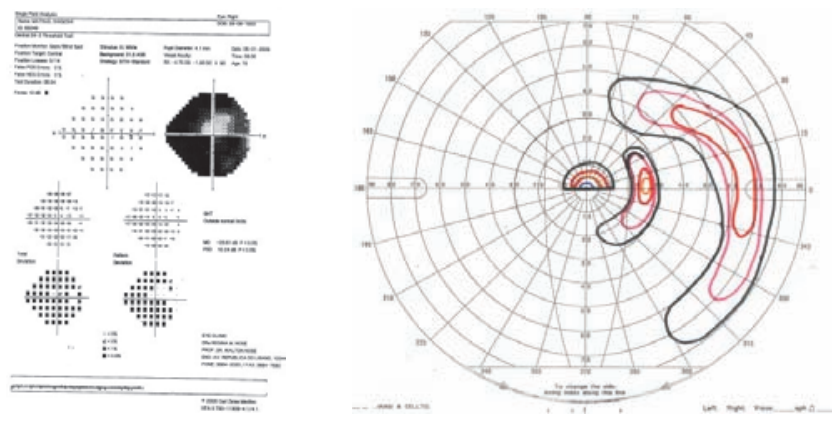

Paciente 1: Observa-se à PAE grande redução da sensibilidade geral, sendo possível observar apenas uma ilha central de visão; Ao exame comPG é possível observar uma ilha central de visão e um campo temporal
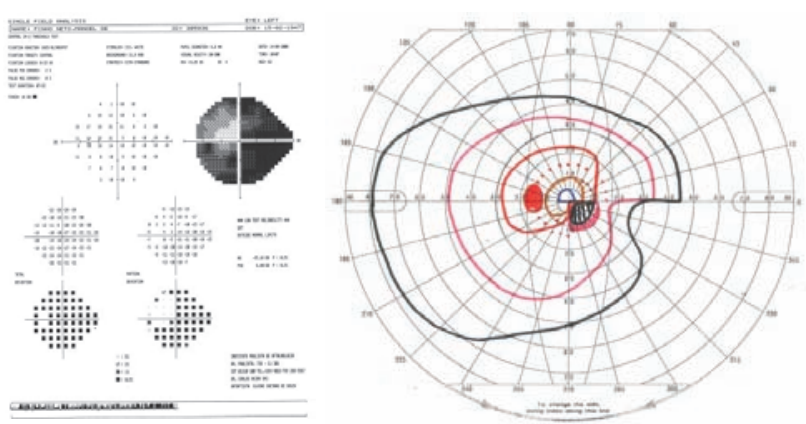

Paciente 3: Observa-se à PAE grande redução da sensibilidade geral com perda importante com campo nasal. Ao exame com PG observa-se um degrau nasal inferior e um escotoma paracentral inferior
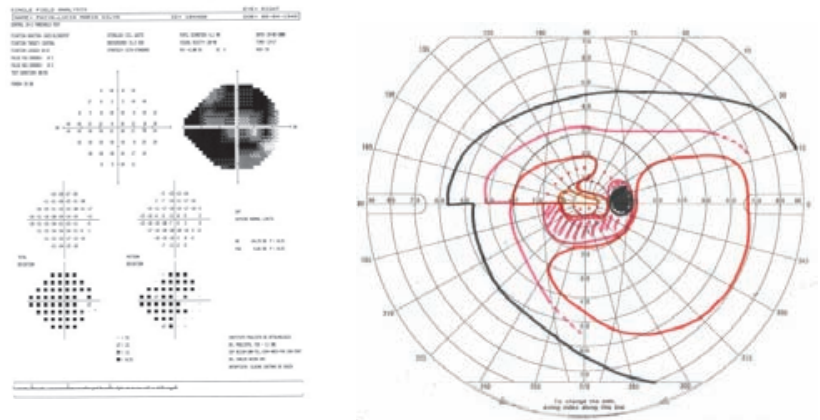

Paciente 5: Observa-se à PAE escotoma arqueado e degrau nasal superior e inferior; Ao exame com PG observa-se degrau nasal inferior e escotoma arqueado inferior
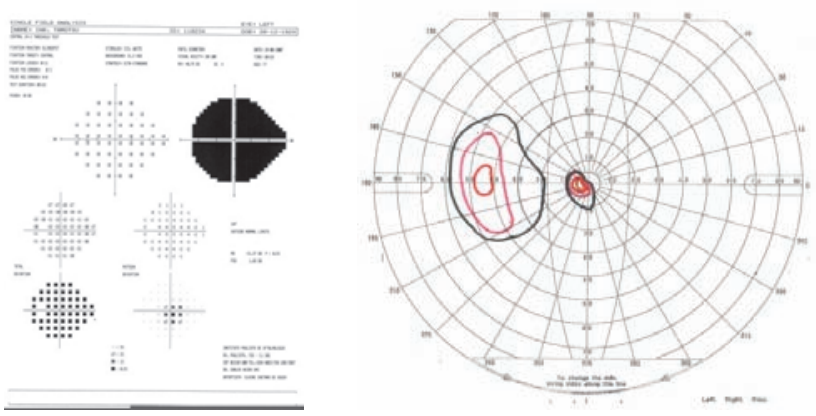

Paciente 2: Observa-se à PAE a presença de um escotoma absoluto. Ao exame com PG observa-se uma ilha central e uma ilha temporal de visão
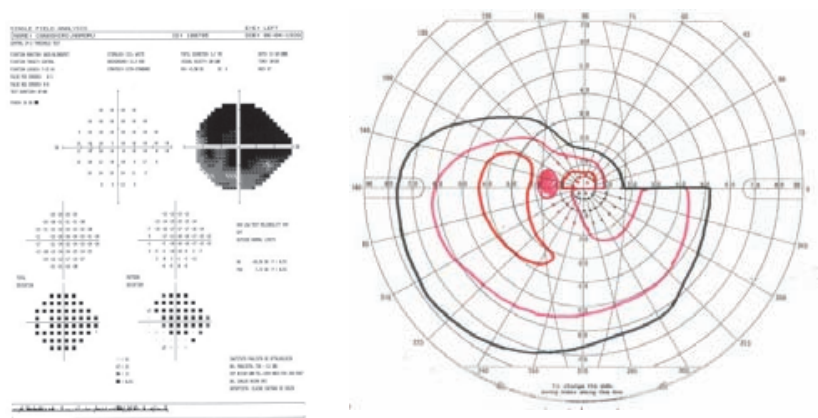

Paciente 4: Observa-se à PAE grande redução da sensibilidade geral com extenso escotoma arqueado superior e degrau nasal; Ao exame com PG observa-se degrau nasal superior e escotoma arqueado superior
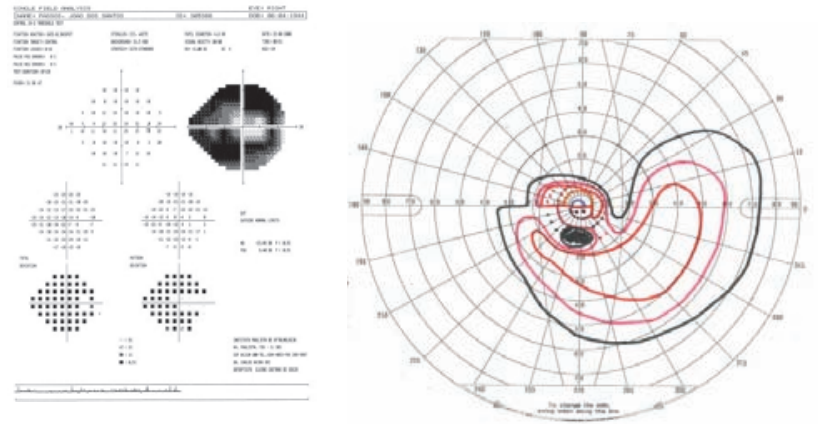

Paciente 6: Observa-se à PAE ilha central de visão; Ao exame com PG observa-se degrau nasal superior e inferior e escotoma paracentral inferior
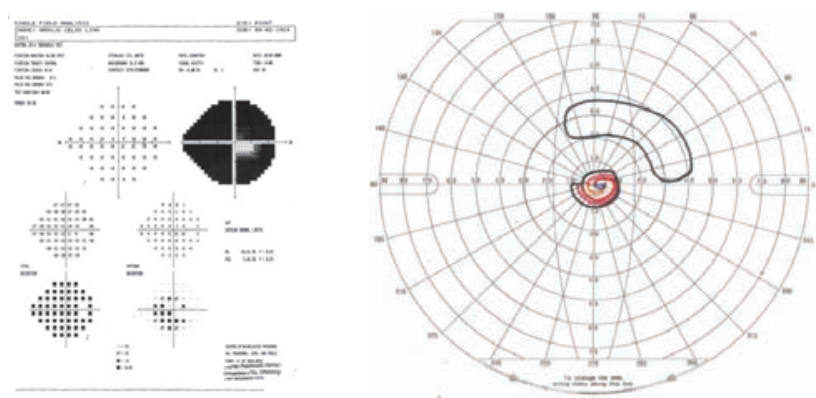

Paciente 7: Observa-se na PAE presença de apenas ilha central de visão;Ao exame com PG observa-se uma ilha central e uma ilha temporal superior de visão 
A PMC com PG é considerada o método de escolha na detecção dos limites de escotomas absolutos bem delimitados ${ }^{(2)}$. Ela possibilita a avaliação de todo o campo visual e quantifica a extensão e formato de defeitos profundos do mesmo. Dessa forma, é ainda um método importante para monitorar progressão, estabilidade ou aumento de defeitos profundos no campo visual de pacientes com campo visual com constrição central ${ }^{(13)}$.

A estratégia limiar central mais utilizada na PAE é a 24-2. Esta estratégia testa os 24 graus centrais da fixação. Em pacientes que apresentam dano campimérico glaucomatoso avançado muitas vezes precisam de estratégias que investiguem uma área maior do campo de visão para que possam ser melhor detectadas ilhas de visão periféricas. Nos pacientes deste estudo, podemos observar ao exame com o perímetro de Goldmnnn ilhas periféricas de visão, principalmente nos casos 1, 2 e 7, que não foram possíveis de se observar ao exame com perímetro de Humprey na estratégia 24-2.

Em um estudo para a avaliação de motoristas de veículos, um número significativo de pacientes com defeitos de campo visual severos, detectados pela PAE, ainda assim mantiveram a habilitação para dirigir após a perimetria cinética ${ }^{(14)}$. Nos pacientes deste estudo, pôde-se observar a presença de um maior número de informações à perimetria de Goldmann em comparação com o resultado encontrado na PAE. Estes achados explicam a capacidade dos pacientes com doença e dano campimétrico ao Humphrey avançados, ainda apresentarem uma capacidade de locomoção surpreendente ao oftalmologista.

A perimetria cinética é frequentemente uma alternativa melhor à perimetria estática quando o defeito campimétrico é extenso e profundo, e o seu formato é importante para diagnóstico e seguimento de alguns pacientes ${ }^{(2)}$. Nos pacientes estudados, podemos observar uma delimitação mais precisa das áreas de escotoma observados na PMC em comparação com as observadas nos gráficos da PAE.

O presente estudo apresenta algumas limitações relacionadas ao pequeno grupo de pacientes observados. Além disso, não foi possível estabelecer uma análise quantitativa para comparar os dois exames pois os mesmos possuem intensidades de estímulos utilizados e extensão do campo estudado diferentes. Desta forma, realizou-se uma análise da extensão dos campos visuais de forma qualitativa.

Na literatura não há um consenso quanto à superioridade de um perímetro em relação ao outro e cada um deles apresenta vantagens e desvantagens distintas, influenciadas pelo estágio da doença e outras variáveis relacionadas à realização do exame ${ }^{(11,15-17)}$. É importan- te ressaltar que o objetivo do trabalho não é comparar os resultados campimétricos obtidos com os dois perímetros, mas apenas demonstrar a importância da perimetria manual e cinética como uma alternativa aos exames computadorizados e estáticos em pacientes com dano glaucomatoso avançado. A PMC mostra-se como uma alternativa valiosa para determinar a extensão real do campo visual destes pacientes e por ser um exame mais fácil e menos cansativo ao paciente quando comparado a estratégias como 10-2, macular e 30-60 do PH.

\section{Conclusão}

A PMC com perímetro de Goldmann mostrou-se útil para complementar a avaliação da real condição campimétrica de pacientes com glaucoma primário de ângulo aberto avançado, submetidos ao exame estático com o perímetro automático de Humphrey.

Este trabalho apresenta um estudo inicial de um pequeno grupo de pacientes. Novos estudos são necessários para complementar os achados encontrados.

\section{Abstract}

Purpose: To evaluate the importance of Goldmann manual kinetic perimetry $(M K P)$ in glaucomatous patients with advanced visual field loss in automated static perimetry (ASP) with Humphrey perimeter (HP). Methods: The MKP with Goldmann perimeter (GP) was performed in patients with advanced primary open angle glaucoma with advanced visual field defect in the ASP with HP. A qualitative analysis was realized on the visual field extension in the Goldmann perimetry of these patients.Results:The results are illustrated in a qualitative way. Conclusion: The MKP with GP was useful to complement the evaluation of the real campimetric condition of advanced primary open angle glaucomatous patients.

Keywords: Perimetry/methods; Kinetics; Glaucoma, open-angle; Visual fields

\section{RefERÊNCIAS}

1. Ramirez AM, Chaya CJ, Gordon LK, Giaconi JA. A comparison of semiautomated versus manual Goldmann kinetic perimetry in patients with visually significant glaucoma. J Glaucoma. 2008;17(2):111-7.

2. Nowomiejska K, Vonthein R, Paetzold J, Zagorski Z, Kardon $\mathrm{R}$, Schiefer U. Comparison between semiautomated kinetic perimetry and conventional Goldmann manual kinetic perimetry in advanced visual field loss. Ophthalmology. 2005;112(8):1343-54. 
3. Anderson DR. Perimetry with and without automation. 2nd ed. St Louis: Mosby; 1987. p. 140.

4. Hudson C, Wild JM. Assessment of physiologic statokinetic dissociation by automated perimetry. Invest Ophthalmol Vis Sci. 1992;33(11):3162-8.

5. Schiefer U, Rauscher S, Paetzold J, Schiller J. Realization of semi-automated kinetic perimetry (SKP) with Interzeag 101 instrument. In: Wall M, Mills RP, editors. Perimetry update. The Hague: Kugler; 2003. p.233-8.

6. Birt CM, Shin DH, Samudrala V, Hughes BA, Kim C, Lee D. Analysis of reliability indices from Humphrey visual field tests in an urban glaucoma population. Ophthalmology. 1997;104(7):1126-30.

7. Szatmáry G, Biousse V, Newman NJ. Can Swedish interactive thresholding algorithm fast perimetry be used as an alternative to goldmann perimetry in neuro-ophthalmic practice? Arch Ophthalmol. 2002;120(9):1162-73.

8. Nevalainen J, Paetzold J, Krapp E, Vonthein R, Johnson CA, Schiefer U. The use of semi-automated kinetic perimetry (SKP) to monitor advanced glaucomatous visual field loss. Graefes Arch Clin Exp Ophthalmol. 2008;246(9):1331-9.

9. Donahue SP. Perimetry techniques in neuro-ophthalmology. Curr Opin Ophthalmol. 1999;10(6):420-8.

10. Katz J, Tielsch JM, Quigley HA, Sommer A. Automated perimetry detects visual field loss before manual Goldmann perimetry. Ophthalmology. 1995;102(1):21-6

11. Beck RW, Bergstrom TJ, Lichter PR. A clinical comparison of visual field testing with a new automated perimeter, the Humphrey Field Analyzer, and the Goldmann perimeter. Ophthalmology. 1985;92(1):77-82.
12. Agarwal HC, Gulati V, Sihota R. Visual field assessment in glaucoma: comparative evaluation of manual kinetic Goldmann perimetry and automated static perimetry. Indian J Ophthalmol. 2000;48(4):301-6.

13. Grover S, Fishman GA, Brown J Jr. Patterns of visual field progression in patients with retinitis pigmentosa. Ophthalmology. 1998;105(6):1069-75.

14. McLean IM, Mueller E, Buttery RG, Mackey DA. Visual field assessment and the Austroads driving standard. Clin Experiment Ophthalmol. 2002;30(1):3-7.

15. Grochowicki M, Vighetto A, Berquet S, Khalfallah Y, Sassolas G. Pituitary adenomas: automatic static perimetry and Goldmann perimetry. A comparative study of 345 visual field charts. Br J Ophthalmol. 1991;75(4):219-21.

16. Stewart WC. Static versus kinetic testing in the nasal peripheral field in patients with glaucoma. Acta Ophthalmol (Copenh). 1992;70(1):79-84.

17. Hotchkiss ML, Robin AL, Quigley HA, Pollack IP. A comparison of Peritest automated perimetry and Goldmann perimetry. Arch Ophthalmol. 1985; 103(3):397-403.

\section{Endereço para correspondência: Nadia Ajub Moysés \\ Rua dos Ingleses, $\mathrm{n}^{\circ} 222$ apto.111 \\ CEP 01329-000 - São Paulo (SP), Brasil \\ E-mail: nadiaajub@gmail.com}

